\title{
Excitation of Geodesic Acoustic Modes by External Fields
}

\author{
K. Hallatschek and G. R. Mckee \\ Max-Planck-Institute for Plasma Physics, EURATOM-IPP Association, Garching, Germany and \\ University of Wisconsin-Madison, Madison, USA
}

(Dated: January 22, 2013)

\begin{abstract}
It is planned to use external magnetic perturbations at acoustic frequencies at the DIII-D tokamak to attempt to drive Geodesic Acoustic Modes (GAM) to modify the turbulent transport. We show that this might not only be possible - despite the well-known electrostatic nature of the GAMs - but a viable and efficient method to generate GAMs in magnetically confined plasmas, by developing an elegant analytic method, which allows to couple numerical dynamic equilibrium calculations to massively parallel non-Boussinesq turbulence code runs and yields practical estimates of the effectivity of the method.
\end{abstract}

Beneficial flows Pressure-gradient driven turbulence in the outer regions of magnetically confined toroidal plasmas drive sheared flows which in turn act to regulate and mediate the saturated state of turbulence and turbulent transport. These turbulence driven global poloidal flows tangential to the iso-pressure surfaces are a focus of research on magnetic plasma confinement experiments [1], since on one hand they generally reduce turbulent convection by shearing vortices apart, and on the other they are an example of spontaneous structure formation in turbulence [2]. Geodesic acoustic modes (GAM), the oscillating flavor of these flows, have been the first to be experimentally confirmed [3] owing to the fact that oscillations are easier to detect than random fluctuations such as the already very early conjectured stationary zonal flows. From the cornucopia of literature on the suppression of convective turbulence by shear flows it appears the fusion problem (largely the achievement of good heat insulation, i.e., confinement) could be facilitated greatly if only the flows could be made strong enough, preferably by external means and not in reliance on the turbulence itself. Unfortunately, injection of momentum into the plasma is generally quite inefficient as the ratio of the power to the momentum injection rate is given by the phase- or particle velocity of the applied waves or particle beams, respectively - both tend to be rather high ( $\gtrsim$ Alfven-speed or multiples of the thermal velocity). Here again the oscillatory property of the GAMs could prove advantageous since in principle they can be generated without momentum input by exploiting the phenomenon of resonance with an oscillating perturbation of the plasma equilibrium. Principal efficiency limitations do not apply when using external magnetic perturbations to do this. In contrast, modulating the pressure by pulsed heating is inherently very inefficient as the power driving the GAMs is a factor $O(\delta T / T)(\delta T / T$ being the relative temperature perturbation of the GAM) smaller than the heating power, as this is the free energy deposited by the heating [4].

The possibility to electromagnetically excite GAMs may appear at first surprising since GAMs are known to be electrostatic in flux tube turbulence studies. Yet, they can interact with magnetic perturbations through global geometry effects. Moreover, the concern that the highly conducting plasma might screen the external magnetic perturbation is unfounded here, since this would require the plasma to be held in place by non-magnetic forces (walls, pressure forces, inertia). In this letter, an elegant framework is described which allows to study the GAM generation by globally solving the perturbed magnetohydrodynamic (MHD) equilibrium equations and (quasi) locally studying the evolution of the turbulence and the resonantly excited GAMs.

Symmetry of perturbation and electromagnetic interaction with GAMs Poloidal flow oscillations arise in a magnetized plasma torus since the magnetic field is inhomogeneous on a magnetic surface $\propto 1 / R(R$ is the major radius), whereas the plasma pressure is constant. Due to the frozen-in magnetic flux, the plasma is compressed/expanded as sketched in fig. 1 through work done by the poloidal motion, which causes a restoring force. An oscillation of frequency $\omega_{G A M} \sim \sqrt{2 \gamma} c_{s} / R=$ $2 \sqrt{2 \gamma p / \rho} / R$ results [5] ( $\gamma \approx 4 / 3$ is the adiabatic index, $c_{s}$ the sound speed, $p$ pressure, $\rho$ the mass density).

As toroidally symmetric (axisymmetric) modes, GAMs only interact with axisymmetric external currents. Only toroidal currents need be considered, since any poloidal

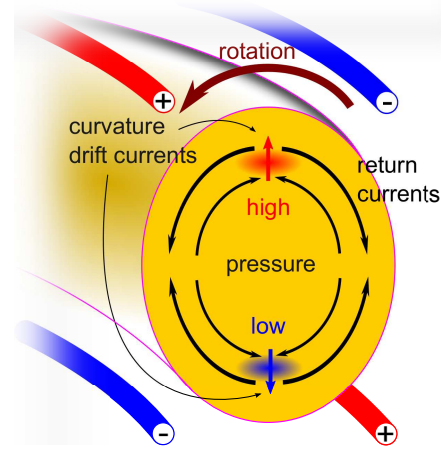

FIG. 1. GAM pressure perturbation, associated poloidal projection of magnetic drift and return currents ("PfirschSchlueter-current"), and external quadrupole current. 
current loop not encircling the plasma column would just produce a field internal to the loop and not affect the plasma. Heuristically, an external quadrupole $(m=2)$ toroidal current (fig. 1) would attract/repulse the toroidal currents within an elliptic plasma column in such a way as to cause a rotation (tilting force) of the column, i.e., act as a source for the GAMs. The current distribution should be up-down and left-right antisymmetric, since otherwise the plasma column is just deformed, not tilted. The corresponding perturbative magnetic field has left-right and up-down symmetry, since it is a pseudo vector.

From a more precise electromagnetic view point, the electric field $\boldsymbol{E}_{e x}=\partial_{t} \boldsymbol{A}_{e x}$ induced by the external perturbation of the vector potential $\boldsymbol{A}_{e x}$ is performing work on the toroidal current $\boldsymbol{j}_{G A M}$ associated with the GAMs, essentially the perturbation of the Pfirsch-Schlueter current caused by the pressure perturbations as sketched in fig. 1. Since these currents have dominant quadrupole symmetry, a quadrupole external current and induced electric field is preferable

Energy conservation implies that said electric work is balanced by the induced electric field due to the GAM integrated over the external current. The interaction is hence due to the weak $O(w / a)$ long range stray fields of the GAM ( $w$ is the radial width of the oscillating region, $a$ the minor plasma radius). In a strictly local flux tube scheme, customary in tokamak turbulence simulations, the radial width of the return current loop of the GAMs is assumed infinitely small, resulting in the neglect of the long range field. Therefore in this framework GAMs cannot be driven by long range magnetic fields and seem purely electrostatic.

Structure of dynamic equilibrium perturbation A quantitative analysis of the resonance requires a global computation for the long range field - so far possible only in MHD - and a more refined plasma description for the turbulence at the resonance. The central unifying idea is to compute the former disregarding inertia and account for the unbalanced inertial forces due to the plasma displacement on the right hand side of the turbulence equations, which are the only GAM source terms in magnetic coordinates.

The (axisymmetric) equilibrium field can be written as

$$
\boldsymbol{B}=\boldsymbol{B}_{\phi}+\nabla \times \boldsymbol{A}_{\phi}, \quad \boldsymbol{A}_{\phi}=\psi \nabla \phi, \quad \boldsymbol{B}_{\phi}=F \nabla \phi
$$

where $\boldsymbol{B}_{\phi}, \boldsymbol{A}_{\phi}$ are the toroidal components of magnetic field and vector potential, $\phi$ is the toroidal angle and $\psi$ the poloidal flux function. Force balance, $\boldsymbol{j} \times \boldsymbol{B}=\nabla p$, then implies $p=p(\psi), F=F(\psi)$ and turns into the Grad Shafranov equation $[6]$

$$
R^{2} \nabla \cdot R^{-2} \nabla \psi+\left(F^{2}\right)^{\prime} / 2+p^{\prime} R^{2}+j_{\phi, e x} R=0,
$$

where the external perturbation current density $j_{\phi, e x}$, whose Lorentz force does not enter the balance, has been made explicit. Restricting the discussion for brevity to the interaction of an up-down symmetric equilibrium with an antisymmetric external current - a good approximation to typical plasma equilibria and linearizing in the perturbation $\delta \psi$ results in

$$
R^{2} \nabla \cdot R^{-2} \nabla \delta \psi+\left(\left(F^{2}\right)^{\prime \prime} / 2+p^{\prime \prime} R^{2}\right) \delta \psi+j_{\phi, e x} R=0,
$$

since then the flux functions $F(\psi), p(\psi)$ are unperturbed due to conserved area/volume enclosed by the flux surfaces. The term $-\left(\left(F^{2}\right)^{\prime \prime} / 2+p^{\prime \prime} R^{2}\right)$, which is essentially proportional to the radial gradient of the toroidal current, quantifies the effects from the presence of the plasma (in contrast to a vaccuum) on the flux perturbation. Since it is negative (hollow current profiles excepted), the plasma usually acts as a paramagnet, amplifying the external fields, basically due to the attraction of parallel currents.

The displacement written as a sum of radial (perpendicular to the flux surface), poloidal and parallel (to $B$ ) components $\boldsymbol{\xi}=\boldsymbol{\xi}_{\text {rad }}+\boldsymbol{\xi}_{\text {pol }}+\boldsymbol{\xi}_{\|}$follows from the conservation of $\psi, p, F$ in the co-moving frame, in other words the three continuity equations

$$
\begin{gathered}
\text { (a) } \delta \psi+\boldsymbol{\xi} \cdot \nabla \psi=0, \quad(b) \quad \nabla \cdot \boldsymbol{\xi}=0, \\
\text { (c) } \nabla \cdot\left[\left(\boldsymbol{\xi}_{\text {rad }}+\boldsymbol{\xi}_{p o l}\right) / R^{2}\right]=0
\end{gathered}
$$

which are just ordinary differential equations in the poloidal angle once $\delta \psi$ is known. The inertial forces $-\rho \partial_{t} \boldsymbol{\xi}$ perpendicular and parallel to the magnetic field can be inserted as the divergence of the ion polarization drift current and a parallel momentum source into customary fluid or gyrokinetic plasma turbulence codes.

The poloidal displacement amplitude of (4), augmented by the resonance quality factor, can serve as an estimate for the GAM amplitude as it is the dominant component for the generation of poloidal rotation. At this point the potentially greatly facilitating effects of the presence of a depression or a null (X-point, saddle point of $\psi$ ) in the magnetic field become apparent. First, due to eq. (4a) the radial displacement amplitude increases inversely proportional to the poloidal field $B_{\text {pol }}=|\nabla \psi| / R$, as visible close to the " $\mathrm{X}$ " on top in the displacement field shown in fig. 2. Inserting all definitions, the maximum radial shift is roughly

$$
\left|\xi_{\text {rad }, \max }\right|=\left|\frac{\delta \psi}{(\nabla \psi)_{\min }}\right| \sim \frac{a \delta B_{\text {rad }}}{2 B_{\text {pol,min }}},
$$

since roughly

$$
\delta \psi=\oint \partial_{p o l} \delta \psi \mathrm{d} l_{p o l}=\oint R \delta B_{r a d} \mathrm{~d} l_{p o l} \sim \frac{a R}{2} \delta B_{r a d}
$$

where $\partial_{p o l} \equiv B_{p o l}^{-1} \boldsymbol{B}_{p o l} \cdot \nabla$ is the poloidal derivative, $l_{p o l}$ the poloidal arc-length, and the factor $1 / 2$ stems from the $m=2$ structure of the perturbation.

Second, the equation for the incompressibility of the toroidal magnetic field (4c) implies that the divergence 


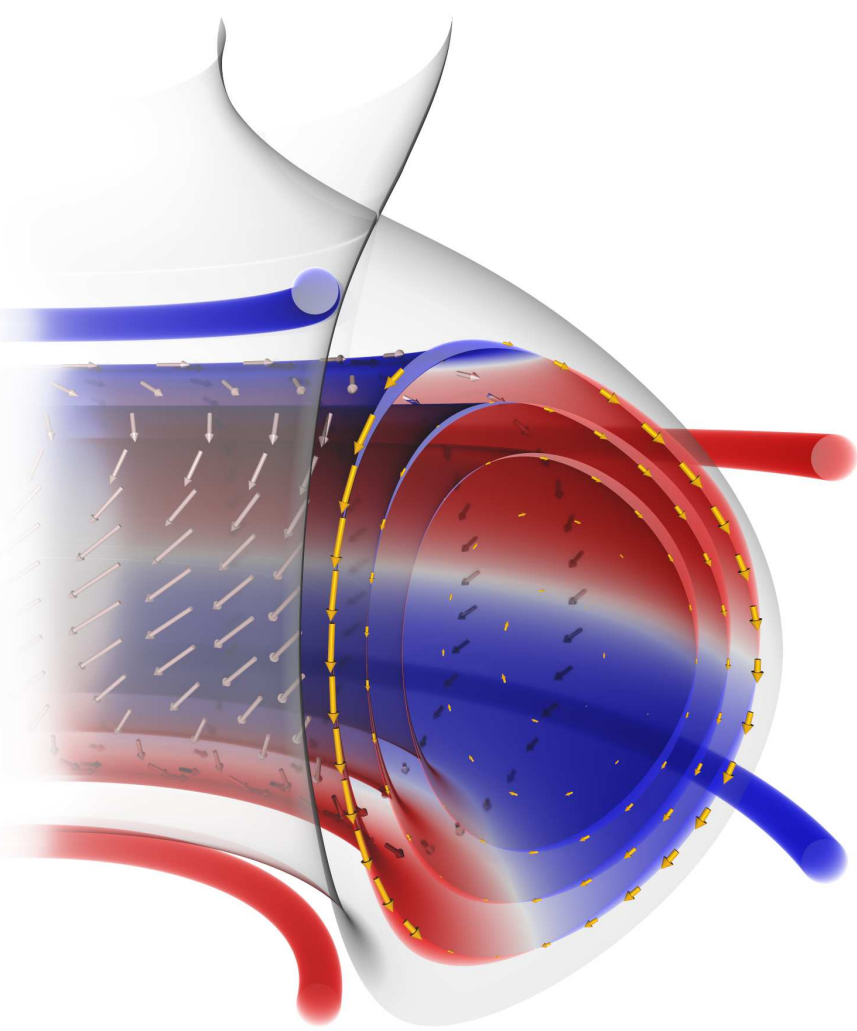

FIG. 2. Linear displacement and flux surface perturbation visualized for hypothetical quadrupole perturbation current $I=90 k A$ ) for DIII-D plasma configuration from discharge \#119527, including the effect of screening currents in the limiter structures (not shown). Gray: unperturbed separatrix. Red/blue toroidal loops: position of positive/negative perturbation current relative to the plasma current. Red/blue shells: volume traversed by positive/negative radial displacement of flux surfaces. Yellow arrows in cross section: poloidal displacement component. White arrows: total displacement tangential to flux surfaces. Flux surfaces are repulsed by negative currents. The radial displacement is particularly strong close to magnetic nulls and to the perturbation current. The resulting poloidal displacement according to the incompressibility condition (4c) is amplified by the nozzle effect of close flux surface spacing at the midplane. For the outermost shown flux surface displacement $\delta B_{\text {pol }} \sim 25 \mathrm{mT},\left|\xi_{\text {rad,max }}\right| \sim 4.8 \mathrm{~cm}$ $\left|\xi_{\text {pol }}\right| \sim 13 \mathrm{~cm}$, nozzle effect $\max (|\nabla \psi|) / \min (|\nabla \psi|) \sim 2.9$ - the displacement diverges at the separatrix.

induced by the radial displacement is balanced by the poloidal shift

$$
\frac{\boldsymbol{\xi}_{p o l}}{R^{2}}=\boldsymbol{B}_{p o l} \oint \frac{\nabla \cdot \frac{\boldsymbol{\xi}_{\text {rad }}}{R^{2}}}{B_{p o l}} \mathrm{~d} l_{p o l} \sim \frac{B_{p o l, \text { max }}}{B_{\text {pol,min }}} \frac{a}{2} \nabla \cdot \frac{\boldsymbol{\xi}_{\text {rad }}}{R^{2}},
$$

which yields another factor proportional to the ratio of local to minimum poloidal field, assuming the dominant radial divergences are placed beneficially at the field minima. Simply speaking, this effect results because the toroidal flux compressed by the shift of neighboring flux surfaces in the vicinity of a magnetic minimum has to "squeeze" through the tighter space between the same flux surfaces at higher poloidal field as indicated, e.g., by the large poloidal displacement components at inboard midplane compared to the radial flux surface shifts in fig. 2). We estimate $\left|\nabla \cdot\left(\boldsymbol{\xi}_{\text {rad }} / R^{2}\right)\right| \sim 2 \xi_{\text {rad }} /\left(a R^{2}\right)$, where the (conservative) factor 2 is again due to $m=2$, and obtain

$$
\xi_{\text {pol,max }} \sim \frac{B_{\text {pol,max }}}{B_{\text {pol,min }}} \xi_{\text {rad }} \sim a \frac{B_{\text {pol,max }} \delta B_{\text {rad }}}{2 B_{\text {pol,min }}^{2}} .
$$

Interaction of turbulence and external induced GAMs To describe the induced resonance layer within a turbulence simulation it is essential to retain the radial variation of the GAM frequency throughout the computational domain, i.e., use a "nonlocal" simulation and not rely on the Boussinesq (flux tube, local) approximation. (In a strictly local framework the whole system oscillates in lockstep, without shearing action on the turbulence.)

Fig. 3 shows the characteristic features in an exemplary electrostatic turbulence run with the NLET code [7] for the reference parameters $\alpha=0.1, \epsilon_{n}=0.05, \eta_{i}=$ $1, \tau=1, q=3, s=1$ at the middle of domain $(r=0)$. The externally induced poloidal displacement was $L_{0} / 2$, ( $L_{0}$ is the ballooning scale length). Typical ballooning scale lengths in a tokamak edge are $0.3 \mathrm{~cm}[8]$. The res-

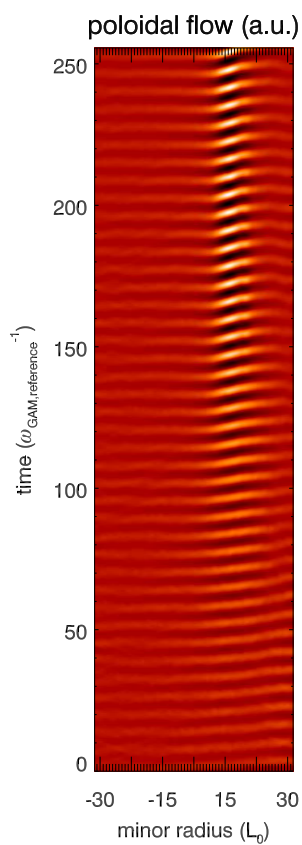

(a)

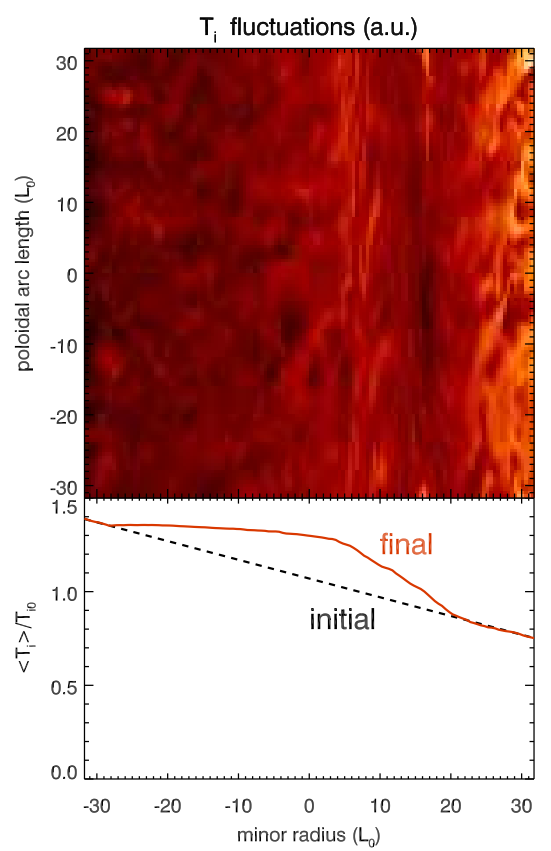

(b)
FIG. 3. GAM excitation in a turbulent nonlocal resistive ballooning scenario in a thin shell at the edge of a circular tokamak discharge; (a) flux surface averaged poloidal flow velocity with resonance, (b) shearing of turbulence fluctuations, top: cut from outboard midplane, bottom: steepening of ion temperature profile. 
onance layer is discernible in the flow profiles (fig. 3a) from the amplitude peaking and the characteristic radial phase variation. The quality factor at the resonance is found to be $Q \sim 25$ (i.e., the externally induced displacement is amplified by a factor $Q$ at the resonance). The sharpness of the resonance depends on the linear and turbulence mediated damping and radial interaction of the GAMs [9, 10].

Here, the peaking and phase variation lead to a strong flow shear at the resonance, and concomitant partial suppression of the turbulence. The partial turbulence quench together with the ensuing profile steepening is followed by a subsequent sharpening of the GAM resonance, which results in a thresholding behavior (fig. 3b) in the forcing and something akin to a transport barrier.

Power requirements The intrinsically required power to generate the GAMs is rather modest for parameters typical for the outer regions of DIII-D or ASDEX Upgrade tokamak discharges. According to the definition of the resonance quality factor $Q$ the power lost by GAM damping is $P_{G A M}=\omega_{G A M} E_{G A M} / Q$. The energy stored in the GAM oscillation is $E_{G A M}=m \omega_{G A M}^{2} d^{2} / 2$, where $d \sim \xi_{\text {pol }} Q$ is the resonance-amplified displacement amplitude, $m=4 \pi^{2} a$ Rwn $_{i}$ the oscillating plasma mass, $w$ the width of the GAM resonance layer, $n$ the ion density and $m_{i}$ the ion mass. For circular slender plasma, the GAM frequency is $\omega_{G A M}=\sqrt{2 \gamma} c_{s} / R, c_{s}=\sqrt{2 T / m_{i}}$. Inserting typical tokamak edge values $T \sim 200 \mathrm{eV} n \sim$ $10^{19} \mathrm{~m}^{-3}, R \sim 1.5 \mathrm{~m}, a \sim 0.5 \mathrm{~m}$, and assuming the width and displacement of the GAMs to be relevant to the turbulence, $w, d \sim 2 \mathrm{~cm}$, and deuterium as medium results in $\omega_{G A M} \sim 175 \mathrm{krad} / \mathrm{s}, E_{G A M}=m d^{2} \omega_{G A M}^{2} / 2 \sim 0.09 \mathrm{~J}$ and $P_{G A M}=E_{G A M} \omega_{G A M} / Q \sim 0.7 \mathrm{~kW}$ for $Q \sim 20$. For realistic tokamak experiments the power requirements are about an order of magnitude smaller, since the observed $\omega_{G A M}$ is reduced compared to the value for a circular plasma by about a factor of two $[5,11]$.

Another loss term results from screening currents in conducting structures of the vessel. With the above parameters as reference, the resistive magnetic skin depth $\lambda=1 / \sqrt{\mu_{0} \omega \sigma} \sim 2 \mathrm{~mm}$ for the specific conductivity of steel $\sigma \sim 1.5 \cdot 10^{6} \mathrm{~S} / \mathrm{m}$. The power flux density into the wall is then $p_{\text {wall }}=\lambda \omega B_{p}^{2} / \mu_{0}$, according to the Poynting theorem. Using eq. (8) it is also possible to estimate the required external perturbation field as $\delta B_{\text {pol }} \sim\left(B_{\text {pol,min }} / B_{\text {pol,max }}\right)^{2}(d / a Q) B_{\text {pol }} \sim 0.07 \mathrm{mT}$, where $B_{p o l} \sim a B /(q R)$ is a reference poloidal field, $q \sim 3$ is the safety factor measuring the pitch of the field lines, $B \sim 2 T$, and $R \sim 1.5 \mathrm{~m}$. The ratio $B_{\text {pol, } \min } / B_{\text {pol, } \max } \sim 2$ in the outer plasma region for standard divertor discharges. Approximating the inner surface of the vessel by a torus with radii $A=1 \mathrm{~m}$ and $R=1.5 \mathrm{~m}$, a negligible total power of $P_{\text {wall }}=p_{\text {wall }} 4 \pi^{2} A R \sim 70 \mathrm{~W}$ results. However, different from the intrinsic losses, this effect depends quadratically on the magnitude of the required perturbation field, quartically on the poloidal field depression and may thus be orders of magnitude larger. Fortunately, it depends on details of the distribution of magnetic field and conducting structures, which can be used for optimization, e.g., by using good conductors close to the perturbation coils to screen less conducting structures.

Conclusions In short the analysis of GAMs generated by external magnetic fields can be split into a global MHD equilibrium problem for the magnetic perturbations and a quasi-local turbulence problem for the resonant layer, which are coupled by inertial forces. Using this idea the required power is estimated to be rather modest for the amplitudes of interest. In contrast, drive schemes based on pulsed external heating or particle or wave momentum are hampered by an inherently very low ratio of free energy or momentum to injected heat.

To achieve GAM amplitudes sufficient to significantly affect the turbulence certainly requires an optimallyconfigured and dedicated setup, which to our knowledge presently is nowhere installed. (Remarkably, even when not sufficient to suppress the turbulence, raising the GAM amplitude may lower the LH transition threshold [12].) However, a first demonstration to measure plasma acoustic resonance spectra may be possible using existing positioning or instability control coils provided they can be driven at the GAM frequency. Within Torkil Jensen Award run time on DIII-D the ELM (edge localized modes) suppression coils (internal, or I-coils) will be used to resonantly excite the GAMs. Switched to $n=0$ configuration they can produce a field of $0.02 \mathrm{mT}$ at $7 \mathrm{kHz}$ yielding a displacement of $\sim 6 \mathrm{~mm}$ under good conditions with the above estimates, while GAM amplitudes $\sim 2 \mathrm{~mm}$ are detectable by spectroscopic imaging and Doppler measurements [13, 14]. The parameters could even be chosen more favorably, e.g., by lowering the equilibrium magnetic field and the GAM frequency.

The optimal conditions for GAM generation can be found by solving eqns. $(3,4)$ for varying plasma shapes, coil and passive conductor positions. Apart from maximizing the displacement at given flux perturbation, the perturbation itself may also be amplified via the second ("un-screening") term in eq. (3). For example elongating the plasma column increases the sensitivity to changes of the vertical magnetic forces (up to the vertical displacement instability). Higher order shaping (indentation) can convert the amplified vertical shift into the desired poloidal displacement.

All things considered, generating GAMs by external fields seems to be an efficient, viable method with potentially far reaching impact, which would allow for the first time to influence poloidal rotation on the fine radial scales relevant to the turbulence. 
1] P.H. Diamond, S.-I. Itoh, K. Itoh, T.S. Hahm, Plasma Phys. Control. Fusion 47, R35 (2005)

[2] K. Hallatschek, et al., Phys. Rev. Lett. 86, 1223 (2001)

[3] M. Jakubowski, R. J. Fonck, G.R. McKee, Phys. Rev. Lett. 89, 265003 (2002)

[4] K. Hallatschek, Phys. Rev. Lett. 93, 125001 (2004)

[5] K. Hallatschek, Plasma Phys. Control. Fusion 49, B137 (2007)

[6] V.D. Shafranov, Sov. Phys. JETP 6, 545 (1958)

[7] K. Hallatschek, et al., Phys. Plasmas 7, 2554 (2000)
[8] A. Zeiler, et al., Phys. Plasmas 3, 2951 (1996)

[9] R. Hager, K. Hallatschek, Phys. Plasmas 16, (2009) 072503

[10] R. Hager, K. Hallatschek, Phys. Plasmas 17, (2010) 032112

[11] G.D. Conway, C. Tröster, et al., Plasma Phys. Control. Fusion 50, 055009 (2008)

[12] G.D. Conway, et al., Phys. Rev. Lett. 106, 065001 (2011)

[13] G. McKee, et al., Phys. Plasmas 10, 1712 (2003)

[14] G.D. Conway, et al., Plasma Phys. Control. Fusion 50, 085005 (2008) 